\title{
Electroacupuncture ameliorates learning and memory in rats with cerebral ischemia-reperfusion injury by inhibiting oxidative stress and promoting p-CREB expression in the hippocampus
}

\author{
RUHUI LIN ${ }^{1}$, YUKUN LIN ${ }^{1,2}$, JING TAO $^{1}$, BIN CHEN $^{3}$, KUNQIANG YU $^{4}$, \\ JIXIANG CHEN ${ }^{4}$, XIAOJIE $\mathrm{LI}^{2}$ and LI-DIAN CHEN ${ }^{1}$ \\ ${ }^{1}$ College of Rehabilitation Medicine; ${ }^{2}$ Fujian Rehabilitation Tech Co-innovation Center; \\ ${ }^{3}$ TCM Rehabilitation Research Center of SATCM; ${ }^{4}$ Fujian Key Laboratory of Exercise Rehabilitation, \\ Fujian University of Traditional Chinese Medicine, Fuzhou, Fujian 350122, P.R. China
}

Received August 15, 2014; Accepted April 17, 2015

DOI: $10.3892 / \mathrm{mmr} .2015 .4321$

\begin{abstract}
The present study aimed to investigate the mechanisms by which electroacupuncture (EA) ameliorates learning and memory in rats with cerebral ischemic-reperfusion $(\mathrm{I} / \mathrm{R})$ injury. Focal cerebral ischemia was induced in adult male Sprague-Dawley (SD) rats by transient middle cerebral artery occlusion (MCAO). Following MCAO surgery, the rats received EA at the Shenting (DU24) and Baihui (DU20) acupoints. The results of the present study demonstrated that treatment with EA significantly ameliorated neurological deficits and reduced cerebral infarct volume $(\mathrm{P}<0.05)$. In addition, EA improved the learning and memory ability of the rats, and markedly activated the cyclic adenosine monophosphate (cAMP) response element-binding protein (CREB) signaling pathway, resulting in the inhibition of cerebral cell apoptosis in the ischemic penumbra. Furthermore, EA increased the activity of superoxide dismutase and glutathione peroxidase, the protein expression levels of phosphorylated-CREB and B-cell lymphoma 2 (Bcl-2), and the mRNA expression levels of Bcl-2. Conversely, EA decreased the levels of malondialdehyde and inhibited the expression levels of Bcl2-associated $X$ protein. The results of the present study suggest that treatment with EA may result in the amelioration of learning and memory ability in rats with cerebral I/R injury.
\end{abstract}

\section{Introduction}

Cognitive function is regarded as the ability to objectively understand ideas. Cognitive function is composed of numerous cognitive domains, including memory, calculation, orientation

Correspondence to: Dr Li-Dian Chen, College of Rehabilitation Medicine, Fujian University of Traditional Chinese Medicine, 1 Huatuo Road, Fuzhou, Fujian 350122, P.R. China

E-mail: nchelidian@yahoo.com

Key words: electroacupuncture, cerebral ischemia-reperfusion, learning and memory, apoptosis, oxidative stress in time and space, structural ability, ability to perform tasks, and language comprehension and application (1-3). Previous studies have suggested that cognitive impairment has a detrimental effect on the recovery of motor function and the ability of impaired patients to perform daily activities, which is an important factor restricting the comprehensive rehabilitation of stroke patients (4-7). Stroke is a leading cause of mortality and permanent disability, and two-thirds of all strokes are considered ischemic (8). In addition, the incidence of cognitive impairment following a stroke may reach $\leq 65 \%$ (9). Furthermore, in $\sim 10$ to $40 \%$ of patients with mild cognitive impairment, the condition may develop into dementia within a year (10-14). There is therefore an urgent requirement for the early detection of cognitive impairment, so that the mental and physical functions of patients can be fully recovered and dementia can be prevented.

Although the pathogeneses of stroke and post-stroke disabilities are complex (15), apoptosis has been suggested as one of the major pathways that may lead to cell death in brain injury following an ischemic stroke (16). In addition, oxidative stress caused by reactive oxygen species (ROS) has long been implicated in neurotoxicity following cerebral ischemic-reperfusion (I/R), and may ultimately result in the initiation of pathways that lead to apoptotic cell death (17). Since free radicals significantly affect the pathogenesis of cerebral ischemic injuries, high levels of free radicals may cause injury to the brain and detrimentally influence nervous function, learning ability, and memory. Therefore, the identification of strategies that suppress, and increase the clearance rate, of free radicals is important in the treatment of ischemic stroke.

The activation of the cyclic adenosine monophosphate (cAMP) response element-binding protein (CREB) transcription factor has been reported to protect neuronal cells in cerebral ischemia $(18,19)$ Furthermore, CREB and the CRE-mediated system are associated with learning and memory, and B-cell lymphoma 2 (Bcl-2) has a pivotal role in the control of cell death. Bcl-2 has been shown to be upregulated by ischemic tolerance, and its expression is regulated by CREB (20). Therefore, activation of CREB phosphorylation can increase 
Bcl-2 expression, which results in protection of the neuronal cells, and ameliorates learning and memory following cerebral ischemia.

Acupuncture is a simple, convenient and cost-effective treatment strategy originating from ancient China, which has been widely used for thousands of years to treat various diseases (21-23). Previous studies have demonstrated the clinical efficacy of acupuncture in stroke and rehabilitation of post-stroke cognitive impairment (24-27). Two acupoints located on the Du meridian; Baihui (DU20) and Shenting (DU24), are considered the most effective locations and have been commonly used in the treatment of cognitive impairment $(28,29)$. In addition, electroacupuncture (EA), which uses fixed frequency and intensity instead of the traditional twisting and extracting techniques, has advantages including stability, strong persistence, and reduced variability and error between practitioners (30). However, the precise mechanism underlying the neuroprotective effects of EA on cognitive impairment remains unclear. Therefore, the present study aimed to evaluate the therapeutic efficacy of EA against post-stroke cognitive impairment. The underlying molecular mechanisms were investigated using a focal cerebral I/R-injured rat model.

\section{Materials and methods}

Animals. Healthy adult male Sprague-Dawley (SD) rats weighing 250-280 g were purchased from the Shanghai SLAC Laboratory Animal Co., Ltd. (Shanghai, China), and housed in pathogen-free conditions with a $12 \mathrm{~h}$ light/dark cycle. All experiments were performed strictly in accordance with the International Ethical Guidelines. The rats had ad libitum access to food and water during the experiment. The present study was approved by the Institutional Animal Care and Use Committee of the Fujian University of Traditional Chinese Medicine (Fuzhou, China).

Establishment of the cerebral I/R-injured rat model. Middle cerebral artery occlusion (MCAO) was used to establish a cerebral I/R-injured rat model, as previously described (31). Briefly, the rats were anesthetized using $10 \%$ chloral hydrate (300 mg/kg; Shanghai Chemical Reagent Co., Ltd., Shanghai, China) injected intraperitoneally. The left common carotid artery, the left external carotid artery, and the internal carotid artery (ICA) were then carefully exposed following a midline neck incision. Approximately 18 to $22 \mathrm{~mm}$ of nylon surgical thread (Beijing Sunbio Biotech Co., Ltd., Beijing, China) was inserted into the ICA until the blunted distal end met resistance, in order to block the left middle cerebral artery (MCA). The thread was removed following $2 \mathrm{~h}$ of occlusion to restore the blood supply to the MCA area, and reperfusion was achieved. Following awakening, the neurological deficit scores of the rats were assessed, prior to their random division into two groups ( $\mathrm{n}=24 /$ group): An ischemia (MCAO) control group, and an $\mathrm{MCAO}+\mathrm{EA}$ group. The rats in the sham group $(\mathrm{n}=24)$ were subjected to the procedure as described above, without the occlusion of the MCA. Following the surgery, the rats were allowed to recover in prewarmed cages.

EA treatment. Following I/R injury, the rats in the EA group received EA treatment. Acupuncture needles, $0.3 \mathrm{~mm}$ in diameter, were inserted at a depth of 2 to $3 \mathrm{~mm}$ into the heads of the rats at the Baihui (DU20) and Shenting (DU24) acupoints. Stimulation was then generated using EA apparatus (model G6805; Suzhou Medical Appliance Factory, Suzhou, China), and the stimulation parameters were set as follows: 5 and $20 \mathrm{~Hz}$ at 1-3 mA, dispersed for $30 \mathrm{~min}$ once daily. The treatment was performed $2 \mathrm{~h}$ following I/R treatment and was continued until the animals were sacrificed by $10 \%$ chloral hydrate intraperitoneal injection and decapitation, 7 days after the operation.

Assessment of neurological deficit scores. The neurological deficit score was assessed in a single-blind manner, as previously described by Chen et al (31). The neurological deficit scores were assessed on the first, third, fifth and seventh day following I/R injury. The scores were determined as follows: Score 0 indicated no neurological deficit; score 1, (failure to fully extend the right forepaw) indicated mild deficits; score 2 , (circling of the right forepaw); score 3, (falling on the right forepaw) indicated moderate deficits; and score 4, (failure to walk) indicated severe deficits. Rats with scores 0 or 4 were excluded from the experiment.

Measurement of cerebral infarct volume. Following completion of the experiment, the rats were sacrificed and their brains rapidly collected. The brain tissue was coronally sectioned into slices $2 \mathrm{~mm}$ thick, prior to being stained with a $2 \%$ solution of tetrazolium chloride (TTC; Sigma-Aldrich, St. Louis, $\mathrm{MO}, \mathrm{USA}$ ) at $37^{\circ} \mathrm{C}$ for $20 \mathrm{~min}$. The sections were subsequently fixed with $4 \%$ paraformaldehyde, as previously described (32). Normal tissue was stained deep red, whereas the infarct area was stained a pale gray color. The stained sections were scanned using a Canon SX20 high-resolution digital camera (Canon, Inc., Tokyo, Japan), and the infarct volume was quantified using the Motic Med 6.0 System (Motic Incorporation, Ltd., Causeway Bay, Hong Kong). The infarct volume was expressed as a percentage of the contralateral hemisphere volume.

Assessment of cognitive function. From the third day following surgery, the spatial learning and memory abilities (33-35) of the rats were investigated by subjecting them to a Morris water maze (Chinese Academy of Sciences, Beijing, China), a circular tank with a diameter of $120 \mathrm{~cm}$ and a height of $50 \mathrm{~cm}$. The tank was filled with $22 \pm 1^{\circ} \mathrm{C}$ water to a depth of $30 \mathrm{~cm}$. A circular escape platform, measuring $6 \mathrm{~cm}$ in diameter and $28 \mathrm{~cm}$ in height, was submerged $2 \mathrm{~cm}$ below the surface of the water. The tank was divided into four quadrants: Northeast, southeast, southwest, and northwest. These points served as the starting positions at which each rat was lowered gently into the water, its head facing the wall of the water maze. Morris water maze tasks include orientation, navigation and space exploration trials. In the first set of trials, each rat was placed in the water at four equidistant locations to the platform. If the rat arrived at the platform within the $90 \mathrm{sec}$ time restriction and remained on it for $3 \mathrm{sec}$, it was considered to have found the platform and was scored on the time taken to complete the task, as well as the length of the chosen route. However, if the rat was unable to find the platform within $90 \mathrm{sec}$, it was placed on the platform for $10 \mathrm{sec}$ and given a time score of 
$90 \mathrm{sec}$. The computer recorded the time taken and the length of the route by which each rat found the safe platform, and each day the average result of the time taken and the length of the route taken for the four quadrants were assessed for each rat. The duration of the first set of trials was 5 days, with the experiment performed once daily.

The second part of the experiment was performed on the seventh day following surgery. Briefly, the ability of each rat to remember the position of the platform was evaluated by measuring the time in which each rat found the platform within the $90 \mathrm{sec}$ time restriction. Following the trials, the rats were thoroughly dried with a hair dryer and returned to their cages.

Determination of superoxide dismutase (SOD) and glutathione peroxidase (GSHPx) activities, and malondialdehyde (MDA) levels. The ischemic brain hippocampus of each rat was collected on the seventh day following MCAO. The brains were rinsed, weighed, and homogenized in 9 volumes of $9 \mathrm{~g} / 1$ ice-cold saline for $10 \mathrm{~min}$ using a Dounce Tissue Grinder (Kimble Chase Life Science and Research Products LLC, Vineland, NJ, USA). The supernatant homogenate was collected following centrifugation at $12,000 \mathrm{x} \mathrm{g}$ for $10 \mathrm{~min}$ at $4^{\circ} \mathrm{C}$. The total protein concentrations were then determined using a Bradford protein assay (Novagen, Inc., Madison, WI, USA). The SOD and GSHPx activities, and the MDA levels were measured using assay kits according to the manufacturer's instructions (Nanjing Jiancheng Bioengineering Institute, Nanjing, China).

Reverse transcription-quantitative polymerase chain reaction $(R T-q P C R)$. The brains of the rats were removed immediately following decapitation, and the ischemic brain tissues were dissected and maintained at $-80^{\circ} \mathrm{C}$ until use. Total RNA was isolated using TRIzol reagent (Invitrogen Life Technologies, Carlsbad, CA, USA), and the Oligo(dT)-primed RNA (1 $\mu \mathrm{g})$ was reverse transcribed into cDNA, according to the manufacturer's instructions (Fermentas, Thermo Fisher Scientific, Pittsburgh, PA, USA). The cDNA was subsequently used to determine the expression levels of Bcl-2 and Bax mRNA by PCR using Taq DNA polymerase (Thermo Fisher Scientific, Inc., Pittsburgh, PA, USA), and $\beta$-actin was used as an internal control. The primer sequence were as follows: Bcl-2, forward, 5'-GGTGGTGGAGGAACTCTTCA-3'; and reverse, 5'-GAGCAGCGTCTTCAGAGACA-3'; Bax forward, 5'-GAGCAGCGTCTTCAGAGACA-3'; and reverse, 5'-TCACGGAGGAAGTCCAGTGT-3'; and $\beta$-actin forward, 5'-ACTGGCATTGTGATGGACTC-3'; and reverse, 5'-CAGCACTGTGTTGGCATAGA-3' (Shanghai Institute of Bioengineering, Shanghai, China). The PCR products were analyzed on a $1.5 \%$ agarose gel and examined using a gel documentation system (Model Gel Doc 2000; Bio-Rad Laboratories Inc., Hercules, CA, USA).

Western blot analysis. The left cerebral hippocampal tissues were collected and triturated in a radioimmunoprecipitation assay buffer (Fansbio, Guangzhou, China), and the proteins were quantified using a bicinchoninic acid assay (Pierce Biotechnology, Inc., Rockford, IL, USA). The protein lysates were separated by electrophoresis by $12 \%$ SDS-PAGE, prior to being transferred onto polyvinylidene difluoride membranes (EMD Millipore, Billerica, MA, USA), which were blocked for $2 \mathrm{~h}$ with $5 \%$ non fat dry milk at room temperature. The blots were then incubated with primary antibodies targeting Bcl-2 (1:1,000; cat. no. 15071; Cell Signaling Technology, Inc., Danvers, MA, USA), Bax (1:1,000; cat. no. 5023; Cell Signaling Technology, Inc.), phosphorylated (p)-CREB (1:1,000; cat. no. 9198; Cell Signaling Technology, Inc.), and $\beta$-actin (1:4,000; cat. no. AA-128; Beyotime Institute of Biotechnology, Haimen, China) overnight at $4{ }^{\circ} \mathrm{C}$, prior to incubation with an appropriate horseradish peroxidase (HRP)-conjugated secondary antibody (1:3,000; goat anti-rabbit IgG; cat. no. 611-1322-0500; Rockland Immunochemicals Inc., Pottstown, PA, USA) for $1 \mathrm{~h}$ at room temperature. The bands were visualized with enhanced chemiluminescence (Amersham, GE Healthcare, Piscataway, NJ, USA), and the images were captured using a Bio-Image Analysis system (Bio-Rad Laboratories Inc.).

Statistical analysis. The data are expressed as the mean \pm standard deviation (SD) and statistically analyzed by one-way analysis of variance using SPSS version 16.0 software (SPSS, Inc., Chicago, IL, USA), prior to being subjected to a post hoc least significant difference test. $\mathrm{P}<0.05$ was considered to indicate a statistically significant difference.

\section{Results}

EA reduces neurological deficits and infarct volume in rats following MCAO. To evaluate whether EA at the Baihui (DU20) and Shenting (DU24) acupoints attenuated ischemic brain injury, the neurological scores were determined at various time points following a stroke. As hypothesized, the rats in the sham group did not exhibit any manifestations of neurological deficits, whereas all of the rats in the MCAO and MCAO + EA groups exhibited clear symptoms of cerebral injury (Fig. 1). However, the neurological function scores were significantly improved in the MCAO + EA group, as compared with the MCAO group $(\mathrm{P}<0.05)$. To further verify these results, the effects of EA on cerebral infarction were evaluated. As shown in Fig. 2, the infarct volume was measured using TTC staining. Normal tissue was stained deep red, whereas the infarct area was a pale cream color. There was a statistically significant decrease in infarct volume in the MCAO + EA group, as compared with the MCAO group $(\mathrm{P}<0.05)$.

EA ameliorates cognitive impairment in cerebral I/R-injured rats. A Morris water maze test was performed on the third to seventh day following MCAO surgery. As shown in Fig. 3, the rats in the MCAO group had longer latency periods and took longer routes to reach the hidden platform. In addition, the number of times that the MCAO rats crossed the location of the platform was significantly lower, as compared with the rats in the sham group $(\mathrm{P}<0.05)$. However, the rats in the EA group had a shorter latency and route length, and the number of times they crossed the platform was higher, as compared with the MCAO group (Fig. 3).

Effects of EA on MDA content and the activity of antioxidant enzymes. To evaluate whether EA affects oxidative stress damage, the MDA content and the activities of antioxidant 


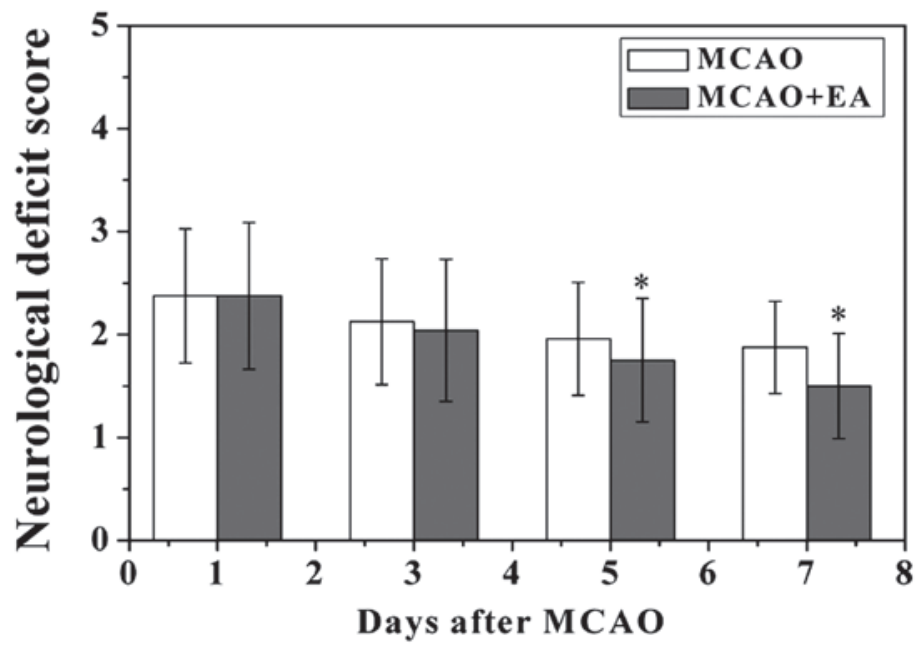

Figure 1. Effects of electroacupuncture (EA) at the Baihui (DU20) and Shenting (DU24) acupoints on the neurological deficits in cerebral ischemia-reperfusion-injured rats. The neurological deficit score was determined, and the data are presented as the mean \pm standard deviation from 18 individual rats. ${ }^{*} \mathrm{P}<0.05$, vs. MCAO group. MCAO, middle cerebral artery occlusion.
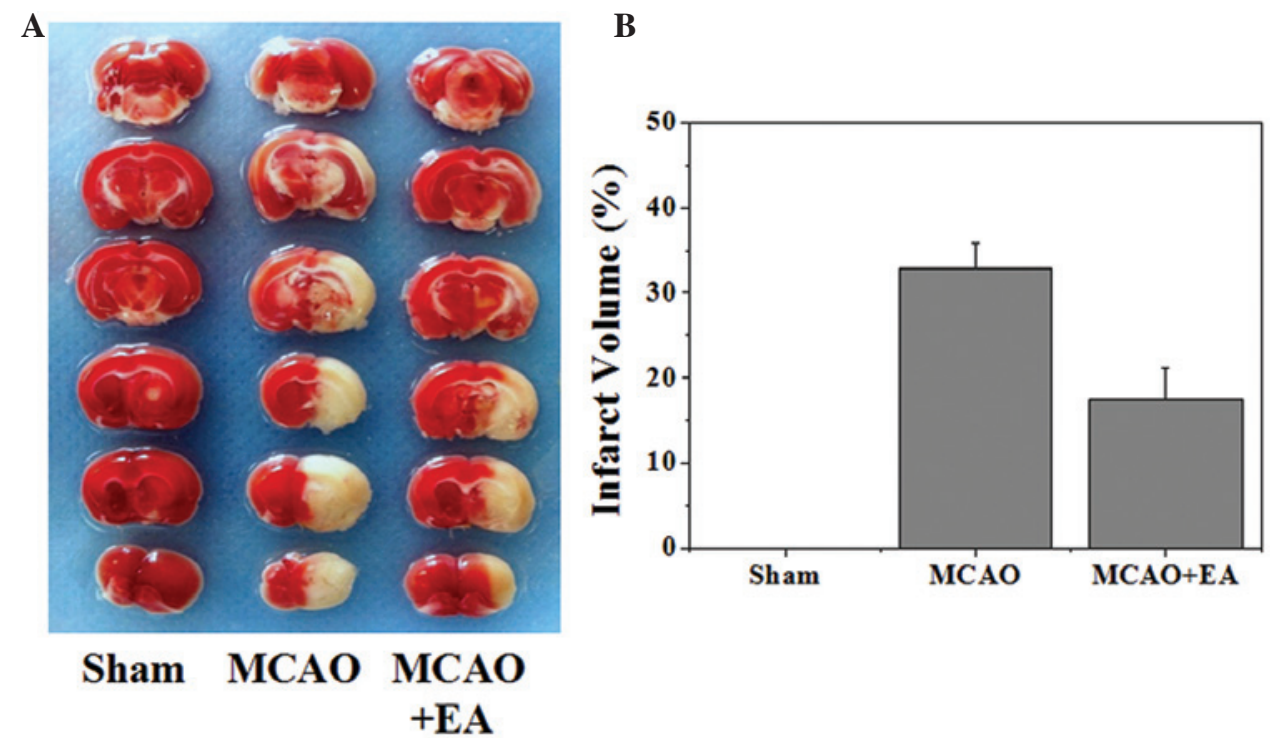

Figure 2. Effects of electroacupuncture (EA) at the Baihui (DU20) and Shenting (DU24) acupoints on cerebral infarction following cerebral ischemia-reperfusion. (A) Tetrazolium chloride staining was used to indicate cerebral infarct volume of Sham, middle cerebral artery occlusion (MCAO) and MCAO + EA groups. The representative captured images were taken using a high-resolution digital camera. (B) Infarct volume was quantified using Motic Med 6.0 system and is represented as a percentage of the total brain volume. Data are presented as the mean \pm standard deviation from six individual rats in each group. ${ }^{*} \mathrm{P}<0.05$, vs. MCAO group.

enzymes in the hippocampus were investigated. MDA content, an index of lipid peroxidation was significantly increased following cerebral I/R injury $(\mathrm{P}<0.05)$, whereas MDA content was significantly decreased after EA treatment, as compared with the MCAO group $(\mathrm{P}<0.05)$. Furthermore, the activities of the antioxidant enzymes SOD and GSHPx were decreased in the MCAO group, as compared with the sham group $(\mathrm{P}<0.05)$. However, EA treatment induced a significant elevation of SOD and GSHPx activities as compared with the MCAO group $(\mathrm{P}<0.05)$ (Fig. 4).

Effects of EA on p-CREB and apoptosis-associated factors. To investigate the mechanism underlying the anti-apoptotic effects of EA, western blot analysis was used to examine the effects of EA on the immunoreactivity of p-CREB in the hippocampus. As shown in Fig. 5, a significant decrease in the immunoreactivity of p-CREB was observed in the hippocampus following MCAO $(\mathrm{P}<0.05)$. Conversely, EA significantly attenuated the decrease in the immunoreactivity of p-CREB $(\mathrm{P}<0.05)$. Western blotting and RT-qPCR were used to evaluate both the protein and mRNA expression levels of the vital target genes Bcl-2 and Bax. As shown in Fig. 6, EA treatment significantly $(\mathrm{P}<0.05)$ increased the mRNA expression levels of Bcl-2 and decreased the mRNA expression levels of Bax caused by the cerebral I/R injury.

\section{Discussion}

EA is a core component of traditional Chinese medicine, which is recognized as an effective treatment for numerous 


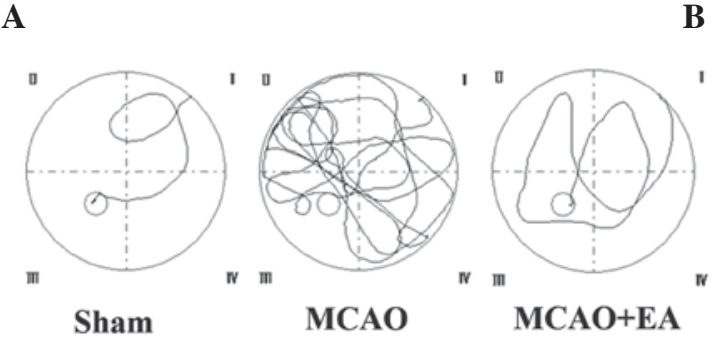

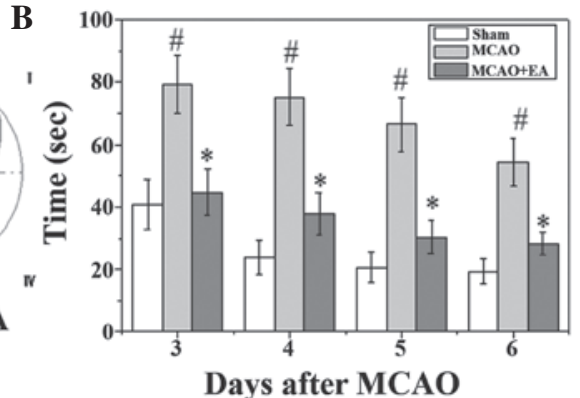

D

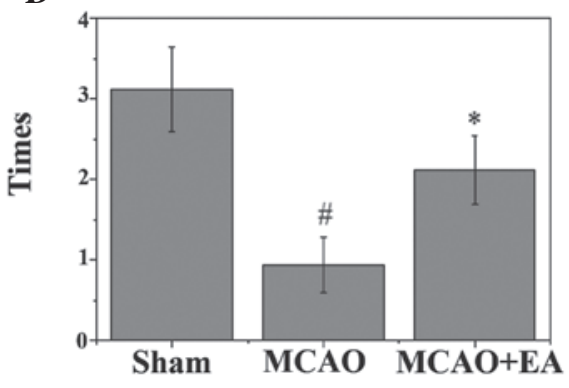

Figure 3. Effects of electroacupuncture (EA) on cognitive impairment in cerebral ischemia-reperfusion (I/R) injured rats. The learning and memory ability of the rats was determined using a Morris water maze test on days 3-6 following I/R injury. (A) Tracing images from the Morris water maze test. (B and C) Time taken for the rats to find the platform (within $90 \mathrm{sec}$ ) and the length of the routes. (D) The number of times the rats passed through the area in which the platform was located was tested on the 7 th day after I/R injury. Data are presented as the mean \pm standard deviation in each group. ${ }^{\#} \mathrm{P}<0.05$, vs. Sham group; ${ }^{*} \mathrm{P}<0 / 05$, vs. MCAO group.

A

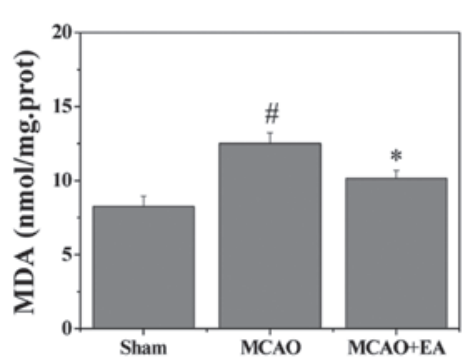

B

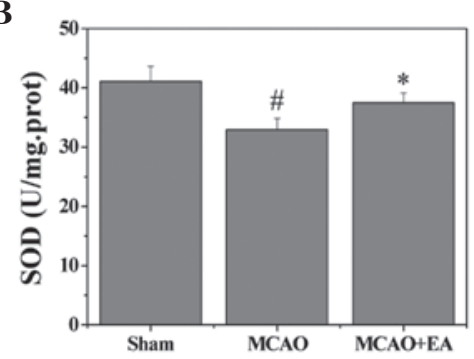

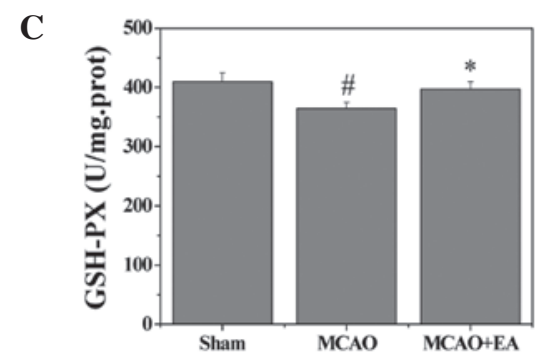

Figure 4. Electroacupuncture (EA) ameliorates oxidative stress following cerebral ischemia-reperfusion (I/R) injury. EA significantly decreased the levels of (A) malondialdehyde (MDA), and increased (B) superoxide dismutase (SOD), and (C) enhanced glutathione peroxidase (GSHPx) activities in I/R rats. Data are presented as the mean \pm standard deviation from six individual rats in each group. ${ }^{\#} \mathrm{P}<0.05$, vs. sham group; ${ }^{*} \mathrm{P}<0.05$, vs. middle cerebral artery occlusion (MCAO) group.

chronic diseases, with no side effects. Numerous studies have demonstrated the clinical efficacy of acupuncture in stroke and cognitive impairment (28). The Baihui (DU20) and Shenting (DU24) acupoints are situated on the Du meridian, which is considered to be beneficial to human health, good spirits, and memory function. The results of the present study demonstrated that EA on the Baihui and Shenting acupoints could significantly ameliorate neurological deficits and reduce cerebral infarct volume. Consistent with previous reports $(27,36,37)$, a Morris water maze test revealed that EA improved the learning and memory ability of rats with cerebral I/R injury, demonstrating the therapeutic efficacy of EA against post-stroke cognitive impairment.

The most effective treatment for acute ischemic stroke is reperfusion of the ischemic penumbra. However, I/R injury often leads to secondary damage. Therefore, anti-reperfusion 

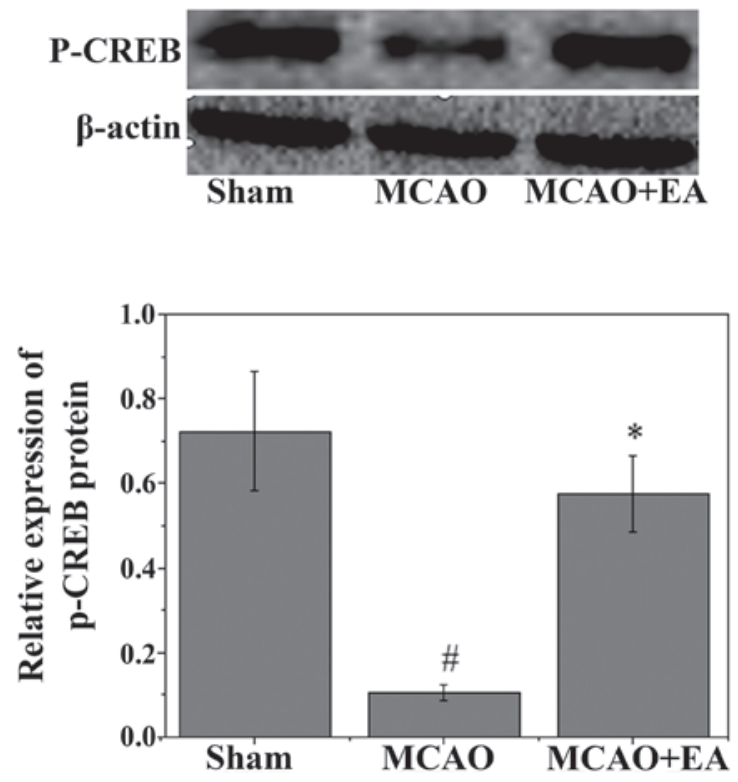

Figure 5. Effects of electroacupuncture (EA) on the expression levels of phosphorylated-cyclic adenosine monophosphate (cAMP) response element-binding protein (p-CREB) in cerebral ischemia-reperfusion-injured rats. The protein expression levels of p-CREB were analyzed by western blotting. $\beta$-actin was used as an internal control for western blotting. The data are representative of six individual rats in each group. Data are presented as the mean \pm standard deviation. ${ }^{\text {"P }}<0.05$, vs. sham group; ${ }^{\text {P }}<0.05$, vs. middle cerebral artery occulsion (MCAO) group.

A
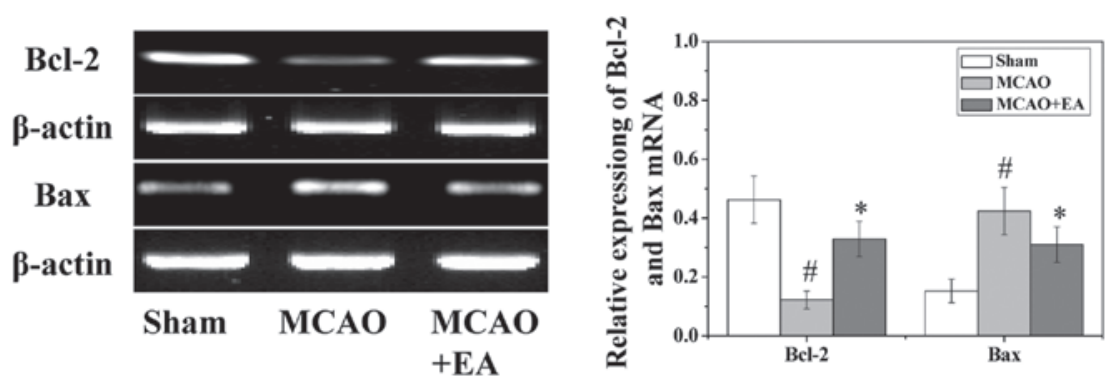

B
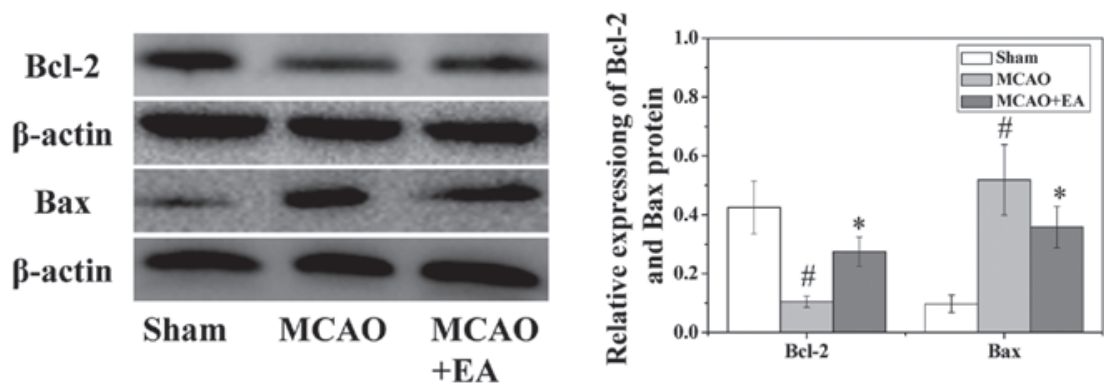

Figure 6. Effects of EA on the expression levels of apoptosis-associated proteins in cerebral ischemia-reperfusion-injured rats. The protein and mRNA expression levels of $\mathrm{B}$ cell lymphoma $2(\mathrm{Bcl}-2)$ and $\mathrm{Bcl}-2$ associated $\mathrm{X}$ protein were determined by (A) reverse transcription quantitative polymerase chain reactionand $(\mathrm{B})$ western blotting 7 days following cerebral ischemia. $\beta$-actin was used as an internal control. The data are representative of six individual rats in each group. Data are presented as the mean \pm standard deviation. ${ }^{*} \mathrm{P}<0.05$, vs. sham group; ${ }^{*} \mathrm{P}<0.05$, vs. middle cerebral artery occlusion $(\mathrm{MCAO})$ group.

injury and neuroprotection are critical for stroke management. Furthermore, oxidative stress has been well-established as the main mechanism underlying $\mathrm{I} / \mathrm{R}$ injury, and reactive oxygen species (ROS) produced in the mitochondria have an important role in regulating the neurocyte apoptotic pathway during $\mathrm{I} / \mathrm{R}(38)$.

Under physiological conditions, ROS are generated at low levels, controlled by endogenous antioxidants, such as SOD and GSHPx (39). However, the sudden overproduction of ROS during cerebral I/R leads to oxidative stress, which results in cell damage in nervous tissue. This may lead to the induction of chain reactions, such as membrane lipid peroxidation (17). ROS produce MDA, a toxic end-product of lipid peroxidation, and MDA levels directly reflect the rate and extent of lipid peroxidation (40). SOD and GSHPx enzymes are thought to act as free radical scavengers that may prevent the deleterious stroke-induced ROS generation (41); therefore, their expression levels may directly reflect the capacity of the brain tissue 
to eliminate free radicals. The results of the present study demonstrated that EA could protect the brain tissue from damage by stimulating SOD and GSHPx activity and by decreasing the levels of MDA.

Apoptosis is one of the predominant types of neurocyte death in the ischemic penumbra during the progression of an ischemic stroke. A previous study (42) demonstrated that memory impairment in MCAO rats was associated with neuronal apoptosis in the hippocampus. In addition, apoptosis was reportedly suppressed by the enhanced expression of Bcl-2 $(43,44)$. The expression of $\mathrm{Bcl}-2$ is mediated by CREB (45), and p-CREB decreases Bax expression (46). In brain tissue, CREB is associated with learning, memory, and dendritic transmission. When hippocampal CREB activity decreases due to cerebral ischemia, impairment of learning and memory ability occurs. Furthermore, CREB knockout mice were reported to exhibit memory impairment (47), with the relative level of CREB activity at the time of learning being a key factor in determining whether a neuron was recruited into the memory trace. The present study demonstrated that compared with the MCAO group, treatment with EA could increase the immunoreactivity of p-CREB and Bcl-2, and decrease the immunoreactivity of Bax. These results suggested that EA is associated with pro-apoptotic activity and the amelioration of learning and memory ability, which may be mediated via activation of CREB phosphorylation.

In conclusion, the present study demonstrated that EA on the Baihui (DU20) and Shenting (DU24) acupoints was able to improve cognitive impairment following cerebral ischemia. The protective effects of EA were associated with an anti-apoptotic mechanism, via activation of CREB, and inhibition of oxidative stress. These results indicate that EA may have therapeutic potential for the treatment of post-stroke cognitive impairment.

\section{Acknowledgements}

The present study was supported by the Mechanism of Acupuncture to Improve Cognitive Function (grant no. X2012 004-collaborative), the National Natural Science Foundation of China (grant no. 81273835), and the National Natural Science Foundation of China (grant no. 81373778). The authors thank Clarity Manuscript Consultants, LLC for editorial assistance with the manuscript.

\section{References}

1. Lindeboom $\mathbf{J}$ and Weinstein $\mathrm{H}$ : Neuropsychology of cognitive ageing, minimal cognitive impairment, Alzheimer's disease, and vascular cognitive impairment. Eur J Pharmacol 490: 83-86, 2004.

2. Nyenhuis Dl, Gorelick PB, Geenen EJ, et al: The pattern of neuropsychological deficits in Vascular Cognitive Impairment-No Dementia (Vascular CIND). Clin Neuropsychol 18: 41-49, 2004.

3. Sachdev PS, Brodaty H, Valenzuela MJ, et al: The neuropsychological profile of vascular cognitive impairment in stroke and TIA patients. Neurology 62: 912-919, 2004.

4. Savva GM and Stephan BC; Alzheimer's Society Vascular Dementia Systematic Review Group: Epidemiological studies of the effect of stroke on incident dementia: A systematic review. Stroke 41: e41-e46, 2010.

5. Liu F, Li ZM, Jiang YJ and Chen LD: A meta-analysis of acupuncture use in the treatment of cognitive impairment after stroke. J Altern Complement Med 20: 535-544, 2014.
6. Li W, Cheng YH and Yu XG: Observation on therapeutic effect of acupuncture combined with medicine on mild cognition disorders in patients with post-stroke. Zhongguo Zhen Jiu 32: 3-7, 2012 (In Chinese).

7. Fang Z, Ning J, Xiong C and Shulin Y: Effects of electroacupuncture at head points on the function of cerebral motor areas in stroke patients: A PET study. Evid Based Complement Alternat Med 2012: 902413, 2012.

8. Lloyd-Jones D, Adams R, Carnethon M, et al; American Heart Association Statistics Committee and Stroke Statistics Subcommittee: Heart disease and stroke statistics - 2009 update: A report from the American Heart Association Statistics Committee and Stroke Statistics Subcommittee. Circulation 119: e21-e181, 2009.

9. Donovan NJ, Kendall DL, Heaton SC, Kwon S, Velozo CA and Duncan PW: Conceptualizing functional cognition in stroke. Neurorehabil Neural Repair 22: 122-135, 2008.

10. Petersen RC, Doody R, Kurz A, et al: Current concepts in mild cognitive impairment. Arch Neurol 58: 1985-1992, 2001.

11. Busse A, Bischkopf J, Riedel-Heller SG and Angermeyer MC: Mild cognitive impairment: Prevalence and incidence according to different diagnostic criteria. Results of the Leipzig Longitudinal Study of the Aged (LEILA75+). Br J Psychiatry 182: 449-454, 2003.

12. Modrego PJ, Fayed N and Pina MA: Conversion from mild cognitive impairment to probable Alzheimer's disease predicted by brain magnetic resonance spectroscopy. Am J Psychiatry 162: 667-675, 2005.

13. Geslani DM, Tierney MC, Herrmann N and Szalai JP: Mild cognitive impairment: An operational definition and its conversion rate to Alzheimer's disease. Dement Geriatr Cogn Disord 19: 383-389, 2005.

14. Ravaglia G, Forti P, Maioli F, et al: Conversion of mild cognitive impairment to dementia: Predictive role of mild cognitive impairment subtypes and vascular risk factors. Dement Geriatr Cogn Disord 21: 51-58, 2006.

15. Nakka VP, Gusain A, Mehta SL and Raghubir R: Molecular mechanisms of apoptosis in cerebral ischemia: Multiple neuroprotective opportunities. Mol Neurobiol 37: 7-38, 2008.

16. Broughton BR, Reutens DC and Sobey CG: Apoptotic mechanisms after cerebral ischemia. Stroke 40: e331-e339, 2009.

17. Manzanero S, Santro T and Arumugam TV: Neuronal oxidative stress in acute ischemic stroke: Sources and contribution to cell injury. Neurochem Int 62: 712-718, 2013.

18. Finkbeiner S: CREB couples neurotrophin signals to survival messages. Neuron 25: 11-14, 2000.

19. Sakamoto K, Karelina K and Obrietan K: CREB: A multifaceted regulator of neuronal plasticity and protection. J Neurochem 116: 1-9, 2011.

20. Meller R, Minami M, Cameron JA, et al: CREB-mediated Bcl-2 protein expression after ischemic preconditioning. J Cereb Blood Flow Metab 25: 234-246, 2005.

21. Zhu LB, Chan WC, Lo KC, Yum TP ad Li L: Wrist-ankle acupuncture for the treatment of pain symptoms: A systematic review and meta-analysis. Evid Based Complement Alternat Med 2014: 261709, 2014.

22. Xie YH, Chai XQ, Wang YL, Gao YC and Ma J: Effect of electro-acupuncture stimulation of Ximen (PC4) and Neiguan (PC6) on remifentanil-induced breakthrough pain following thoracal esophagectomy. J Huazhong Univ Sci Technology Med Sci 34: 569-574, 2014.

23. Hyun SH, Im JW, Jung WS, et al: Effect of ST36 acupuncture on hyperventilation-induced $\mathrm{CO}_{2}$ reactivity of the basilar and middle cerebral arteries and heart rate variability in normal subjects. Evid Based Complement Alternat Med 2014: 574986, 2014.

24. Zhao L, Zhang FW, Zhang H, et al: Mild cognitive impairment disease treated with electroacupuncture: A multi-center randomized controlled trial. Zhongguo Zhen Jiu 32: 779-784, 2012 (In Chinese).

25. Zhou L, Zhang YL, Cao HJ and $\mathrm{Hu} \mathrm{H}$ : Treating vascular mild cognitive impairment by acupuncture: A systematic review of randomized controlled trials. Zhongguo Zhong Xi Yi Jie He Za Zhi 33: 1626-1630, 2013 (In Chinese).

26. Zhang H, Zhao L, Yang S, et al: Clinical observation on effect of scalp electroacupuncture for mild cognitive impairment. J Tradit Chin Med 33: 46-50, 2013.

27. Li X, Guo F, Zhang Q, et al: Electroacupuncture decreases cognitive impairment and promotes neurogenesis in the APP/PS1 transgenic mice. BMC Complement Altern Med 14: 37, 2014. 
28. Zhao L, Zhang H, Zheng Z and Huang J: Electroacupuncture on the head points for improving gnosia in patients with vascular dementia. J Tradit Chin Med 29: 29-34, 2009.

29. Chen LP, Wang FW, Zuo F, Jia JJ and Jiao WG: Clinical research on comprehensive treatment of senile vascular dementia. J Tradit Chin Med 31: 178-181, 2011.

30. Zhou F, Guo J, Cheng J, Wu G and Xia Y: Electroacupuncture increased cerebral blood flow and reduced ischemic brain injury: Dependence on stimulation intensity and frequency. J Appl Physiol 111: 1877-1887, 2011

31. Chen A, Lin Z, Lan L, et al: Electroacupuncture at the Quchi and Zusanli acupoints exerts neuroprotective role in cerebral ischemia-reperfusion injured rats via activation of the PI3K/Akt pathway. Int J Mol Med 30: 791-796, 2012.

32. Lan L, Tao J, Chen A, et al: Electroacupuncture exerts anti-inflammatory effects in cerebral ischemia-reperfusion injured rats via suppression of the TLR $4 / \mathrm{NF}-\kappa \mathrm{B}$ pathway. Int J Mol Med 31: 75-80, 2013.

33. Pouzet B, Zhang WN, Feldon J and Rawlins JN: Hippocampal lesioned rats are able to learn a spatial position using non-spatial strategies. Behav Brain Res 133: 279-291, 2002.

34. Veng LM, Granholm AC and Rose GM: Age-related sex differences in spatial learning and basal forebrain cholinergic neurons in F344 rats. Physiol Behav 80: 27-36, 2003.

35. Feng X, Yang S, Liu J, et al: Electroacupuncture ameliorates cognitive impairment through inhibition of NF- $\kappa \mathrm{B}$-mediated neuronal cell apoptosis in cerebral ischemia-reperfusion injured rats. Mol Med Rep 7: 1516-1522, 2013.

36. Cheng H, Yu J, Jiang Z, et al: Acupuncture improves cognitive deficits and regulates the brain cell proliferation of SAMP8 mice. Neurosci Lett 432: 111-116, 2008.

37. Lu B, Ma Z, Cheng F, et al: Effects of electroacupuncture on ethanol-induced impairments of spatial learning and memory and Fos expression in the hippocampus of rats. Neurosci Lett 576: 62-67, 2014
38. Sun L, Jin Y, Dong L, Sumi R, Jahan R and Li Z: The neuroprotective effects of Coccomyxa gloeobotrydiformis on the ischemic stroke in a rat model. Int J Biol Sci 9: 811-817, 2013.

39. Loh KP, Huang SH, De Silva R, Tan BK and Zhu YZ: Oxidative stress: Apoptosis in neuronal injury. Curr Alzheimer Res 3: 327-337, 2006.

40. Springer JE, Azbill RD, Mark RJ, Begley JG, Waeg G and Mattson MP: 4-hydroxynonenal, a lipid peroxidation product, rapidly accumulates following traumatic spinal cord injury and inhibits glutamate uptake. J Neurochem 68: 2469-2476, 1997.

41. Niizuma K, Yoshioka $\mathrm{H}$ and Chen $\mathrm{H}$, et al: Mitochondrial and apoptotic neuronal death signaling pathways in cerebral ischemia. Biochim Biophys Acta 1802: 92-99, 2010.

42. Li X, Han F, Liu D and Shi Y: Changes of Bax, Bcl-2 and apoptosis in hippocampus in the rat model of post-traumatic stress disorder. Neurol Res 32: 579-586, 2010.

43. Franke C, Noldner M, Abdel-Kader R, et al: Bcl-2 upregulation and neuroprotection in guinea pig brain following chronic simvastatin treatment. Neurobiol Dis 25: 438-445, 2007.

44. Sriraksa N, Wattanathorn J, Muchimapura S, Tiamkao S, Brown K and Chaisiwamongkol K: Cognitive-enhancing effect of quercetin in a rat model of Parkinson's disease induced by 6-hydroxydopamine. Evid Based Complement Alternat Med 2012: 823206, 2012.

45. Kim SS, Jang SA and Seo SR: CREB-mediated Bcl-2 expression contributes to RCAN1 protectionfrom hydrogen peroxide-induced neuronal death. J Cell Biochem 114: 1115-1123, 2013.

46. Royer C, Lucas TF, Lazari MF and Porto CS: 17Beta-estradiol signaling and regulation of proliferation and apoptosis of rat Sertoli cells. Biol Reprod 86: 108, 2012.

47. Han JH, Kushner SA, Yiu AP, et al: Neuronal competition and selection during memory formation. Science 316: 457-460, 2007. 